\title{
ARTICLE
}

Acute lymphoblastic leukemia

\section{Progress against childhood and adolescent acute lymphoblastic leukaemia in the Netherlands, 1990-2015}

\author{
Ardine M. J. Reedijk $\mathbb{1}^{1} \cdot$ Jan Willem W. Coebergh ${ }^{2} \cdot$ Hester A. de Groot-Kruseman $^{3} \cdot$ Inge M. van der Sluis $^{1}$ • \\ Leontien C. Kremer ${ }^{1} \cdot$ Henrike E. Karim-Kos $\mathbb{B}^{1,4} \cdot$ Rob Pieters $^{1,3}$
}

Received: 11 June 2020 / Revised: 29 July 2020 / Accepted: 6 August 2020 / Published online: 21 August 2020

(c) The Author(s) 2020. This article is published with open access

\begin{abstract}
We assessed the epidemiologic progress against childhood and adolescent acute lymphoblastic leukaemia (ALL) in the Netherlands over a 26 year period. ALL patients $<18$ years were selected from the Netherlands Cancer Registry and the Dutch Childhood Oncology Group. Trend analyses were performed over time and by age group and ALL subtype. Between 1990 and 2015, 2997 ALL patients were diagnosed, i.e. 115 patients (range 87-147) per year. Overall incidence remained stable at 37 per million children, despite increases for B-cell precursor ALL (BCP-ALL) at age 10-14 years (AAPC $+1.4 \%$, $p=0.04$ ) and T-cell ALL at $15-17$ years (AAPC $+3.7 \%, p=0.01$ ). Five-year survival increased from $80 \%$ in $1990-94$ to $91 \%$ in $2010-15(p<0.01)$. Mortality decreased by $4 \%$ annually $(p<0.01)$. Patients $15-17$ years were increasingly treated in a paediatric oncology centre, from 35\% in 1990-94 to 87\% in 2010-15 and experienced a 70\% reduction of risk of death compared to those treated outside such a centre $(p<0.01)$. Significant progress against childhood ALL has been made in the Netherlands, visible by improved survival rates coinciding with declining mortality rates. These outcomes were accompanied by stable incidence rates, despite increases for BCP-ALL at age 10-14 years and T-cell ALL at age $15-17$ years.
\end{abstract}

\section{Introduction}

Increases in incidence of childhood acute lymphoblastic leukaemia (ALL) have been reported at the beginning of the 21 st century [1-5]. No clear explanations for these increases could be given in the absence of specific causes. ALL is the most common cancer among children, as well as the most

These authors contributed equally: Henrike E. Karim-Kos, Rob Pieters

Supplementary information The online version of this article (https:// doi.org/10.1038/s41375-020-01024-0) contains supplementary material, which is available to authorized users.

Henrike E. Karim-Kos

h.e.karim-kos@prinsesmaximacentrum.nl

1 Princess Máxima Center for Pediatric Oncology, Utrecht, The Netherlands

2 Department of Public Health, Erasmus University Medical Center, Rotterdam, The Netherlands

3 Dutch Childhood Oncology Group, Utrecht, The Netherlands

4 Department of Research, Netherlands Comprehensive Cancer Organisation (IKNL), Utrecht, The Netherlands frequent cause of death from cancer below the age of twenty $[6,7]$. Incidence and mortality trends are summary measures that provide snapshots of a long-term, time-dependent process [8]. Recent population-based studies for paediatric ALL focusing on incidence and mortality are limited in literature and are lacking for the Netherlands.

Since the early 1970s, treatment of children with ALL has been organised with national treatment protocols in the Netherlands. At that time the Dutch Childhood Leukemia Study Group (DCLSG, since 2002 extended to the Dutch Childhood Oncology Group [DCOG]) was established. The DCLSG/DCOG has a trial and data centre, with a reference diagnostic laboratory for leukaemias, and it also coordinates clinical trials, since 2003 also for solid tumours. Most recent changes in therapy were improvements in chemotherapy and better ways to stratify patients to receive less or more intensive therapy [9-12]. Trends in childhood ALL survival have been published in relation to therapeutic developments in several European countries, Japan and the US [13]. Both Pastore et al. [14] and Stiller et al. [15] have examined that changes in population-based survival parallel those reported by the relevant clinical trials. The increasing level of participation in trials, facilitated by the organisation of 
specialised care, has underpinned the substantial improvements in survival seen at the population level [15].

The overall aim of this study was to assess the progress made for children and young adolescents with ALL in the Netherlands since 1990 by analysing trends in incidence and survival against the background of subsequent treatment regimens. Data from the Netherlands Cancer Registry (NCR) were combined with detailed leukaemia and treatment characteristics from the DCOG registry. Mortality data on cause of death were derived from the website of Statistics Netherlands. In addition, detailed analyses were made regarding ALL subtype and site of treatment.

\section{Patients and methods}

\section{Study population}

Patients aged $<18$ years and diagnosed with ALL (ICD-O-3 M9811-9818 and M9835-9837) from January 1990 to December 2015 were extracted from the NCR. For completeness a linkage with DCOG was performed and after this linkage the ALL subtype, site of treatment and treatment protocol could be determined for patients known at the DCOG registry. A total of 2947 patients with ALL from the NCR were linked with 2882 patients from the DCOG, yielding 2997 patients eligible for inclusion (Supplementary Fig. 1). In case of discrepancies in morphology, DCOG data were preferred over NCR data because of their role as a reference laboratory. For patients ascertained in the NCR only, morphology codes (according to the International Classification of Diseases for Oncology (ICD-O)) as registered in the NCR were taken. ALL may be of B-cell precursor (BCP) or T-cell (T-cell) lineage [16]. For 11 patients $(<1 \%)$ the subtype was unknown.

\section{The Netherlands Cancer Registry}

The nationwide population-based NCR is maintained and hosted by the Netherlands Comprehensive Cancer Organisation (IKNL) and has a national coverage since 1989 with a completeness of at least $96 \%$ of all newly diagnosed malignancies in the Netherlands [17]. The NCR is notified by the Nationwide Network and Registry of Histopathology and Cytopathology, and the National Registry of Hospital Discharges. Retrospectively, data is extracted on patient, tumour and treatment characteristics. Primary therapy started within 9 months after diagnosis is recorded following order of administration and includes radiotherapy, systemic chemotherapy, and stem cell transplantation (SCT). Information on vital status (alive, dead, or emigration) is obtained by annual linkage of the NCR with the Nationwide Population Registries Network that holds vital statistics on all residents in the Netherlands. Last linkage was at February 1 st 2019.

\section{Registry of the Dutch Childhood Oncology Group}

The centrally reviewed results of bone marrow, peripheral blood and spinal fluid samples taken at diagnosis are registered at the DCOG database. ALL diagnosis is based on a combination of cytomorphology, immunophenotyping and -increasingly- (molecular) cytogenetics [12]. Baseline patient and leukaemia characteristics (e.g., sex, age, white blood cell count at diagnosis, pre-existing syndromes and cytogenetics) are collected from the treating hospitals and included in the database. Eligibility and inclusion in specific clinical trials or treatment protocols are centrally registered at the DCOG. For these "in-trial patients" details regarding diagnosis, treatment, response to treatment, toxicity and outcome including relapse(s), second malignancy, and death were also registered. Five consecutive ALL treatment protocols (ALL7 - ALL11) were active during our study period [9-12], plus specific protocols for infants, patients aged <1 year, since 1999 (Interfant) [18, 19] and for Philadelphia-chromosome-positive ALL (EsPhALL) since 2005 [20] (Supplementary Fig. 2). Only patients treated in the seven paediatric oncology centres in the Netherlands were included in the DCOG registry. In the 1990s treatment was also performed in some non-university hospitals, under supervision of one of the paediatric centres. For the site of treatment analyses patients were considered as being treated outside a paediatric oncology centre if they were unknown in the DCOG registry.

\section{Mortality data}

Disease-specific mortality rates from 1980 to 2016 were derived from Statistics Netherlands (CBS). Because of privacy regulations, linkage between the NCR and CBS is not allowed in the Netherlands on a routine base. The lymphoid leukaemia (LL) specific ICD-9 code "204" and ICD-10 code "C91" were used to identify the number of persons who died from LL. Mortality data by age at death were presented by 5 -year age groups (i.e., $0-4,5-9,10-14$, and $15-19$ years).

\section{Statistical analyses}

Characteristics of the study population were described as percentages in relation to the following five periods of diagnosis: 1990-94, 1995-99, 2000-04, 2005-09, and 2010-15. Differences among categorical variables were tested with the $\chi 2$ tests.

Annual incidence and mortality rates were calculated per million person years, using the annual mid-year population 
size as obtained from Statistics Netherlands. Rates were age-standardised according to the age structure of the World standard population for age ranges $0-14$ year, 0-17 year for estimation of incidence rates, and 0-19 year for mortality rates [21]. Linear regression modelling assessed trends over time (i.e. time period 1990-2015 for incidence and time period 1980-2016 for mortality). A regression line was fitted to the natural logarithm of the incidence and mortality rates, including calendar year as a continuous variable [21]. Results were reported as average annual percent changes (AAPC) along with the corresponding 95\% confidence interval (CI) and $p$ values.

Survival time was calculated as the time elapsed between the date of diagnosis and the date of death due to any cause (event) or date at last follow-up (i.e. alive or censored). Traditional actuarial survival analysis was used to calculate overall survival (OS) at 5 and 10 years after diagnosis. Changes over time in observed 5-and 10-year survival were evaluated with a p-trend analysis for period of diagnosis, sex, age at diagnosis, ALL subtype, and site of treatment by using parametric survival models (streg), adjusted for follow-up time (in years). To evaluate their effect on the risk of dying per period of diagnosis, these parameters were entered in a multivariable analysis model. For survival analyses according to treatment protocol, patients eligible and treated according to the protocol were included (DCOG patients only).

All analyses were performed with STATA/SE 14.1 (StataCorp LP, College Station, Texas, USA). Joinpoint regression program (version 4.5.0.1) was used to check for incidence trend transitions during the study period [22]. A $p$ value $<0.05$ was considered statistically significant.

\section{Role of the funding source}

The funding source had no role in the study design, data collection, analyses and interpretation of the results, nor in writing of this manuscript.

\section{Results}

\section{Patient and leukaemia characteristics}

Between 1990 and 2015, 2997 children and adolescents aged $<18$ years were diagnosed with ALL in the Netherlands and analysed in this study. The majority of patients had a diagnosis confirmed by the reference laboratory of the DCOG (96\%). Median age at diagnosis was 5 years (interquartile range 3-9 years). More boys than girls were diagnosed with ALL (male to female ratio (M:F ratio) being 1.4) (Table 1). Patients below five years were mainly diagnosed with BCP-ALL (94\%), decreasing with age to $73 \%$ of the patients aged $15-17$ years.

Over time patients were increasingly treated at a paediatric oncology centre, $94 \%$ in the period 1990-94 compared to $98 \%$ in the period 2010-15 $(p<0.01)$ (Table 1). In the last period, $2010-15$, only 16 patients were not known in a DCOG centre because of treatment abroad $(n=4)$, treatment at an adult ward $(n=9)$ or death at first presentation at a hospital $(n=3)$.

\section{Trends in incidence rates}

On average, 115 patients (range 87-147) were diagnosed with ALL annually. The world standardised incidence rate for patients aged $0-17$ years (WSR 0-17) increased over time by $0.6 \%$ per year $(p=0.05)$, from 30 per million person-years in 1990-94 to 37 in 2010-15. This increase did not pertain to any age group (Fig. 1) or gender (Supplementary Table S1). BCP-ALL increased over time by $0.6 \%$ per year $(p=0.06)$, from 26 per million person-years in 1990-94 to 32 in 2010-15 (Supplementary Table S1). However, for patients aged 10-14 years the increase was significant (AAPC $+1.4 \%, p=0.04$ ). T-cell ALL only showed an increasing trend for young adolescents (15-17 years) from two patients per year on average in the 1990s to four in 2010-15 (AAPC $+3.7 \%, p=0.01)$ (Supplementary Table S1).

\section{Trends in overall survival}

Five-year overall survival increased from $80 \%$ (SE 2\%) in 1990-94 to $91 \%$ (SE 1\%) in 2010-15 ( $p<0.01)$ (Fig. 2). Ten-year overall survival increased from $76 \%$ (SE $2 \%$ ) in 1990-94 to 87\% (SE 1\%) in 2005-09 ( $p<0.01)$ (Fig. 2). Five-year survival significantly increased for infants aged $<1$ year from $27 \%$ in $1990-99$ to $66 \%$ in $2000-15$ ( $p<$ 0.01 ); for patients aged $1-4$ years from $86 \%$ in $1990-94$ to 95\% in 2010-15 $(p<0.01)$; for patients aged 5-9 years from $86 \%$ in $1990-94$ to $96 \%$ in $2010-15(p<0.01)$; for patients aged $10-14$ years from $72 \%$ in $1990-94$ to $85 \%$ in 2010-15 $(p<0.01)$; for patients aged 15-17 years from $57 \%$ in $1990-94$ to $74 \%$ in $2010-15(p=0.02)$ (Fig. 3a and Supplementary Table S2). The 10-year overall survival did also increase significantly for all age groups, only nonsignificantly for patients aged $10-14$ years. (Fig. 3b and Supplementary Table S2).

Five-year overall survival significantly increased for both boys and girls; for boys from $75 \%$ in $1990-94$ to $90 \%$ in $2010-15(p<0.01)$; for girls from $86 \%$ to $91 \%(p=0.04)$ (Supplementary Table S2). Ten-year overall survival also significantly increased for boys, from $72 \%$ in $1990-94$ to $89 \%$ in $2005-09(p<0.01)$. Five- and 10 -year overall survival significantly increased for BCP-ALL, from $81 \%$ in 
Table 1 Patient characteristics of patients aged < 18 years with acute lymphoblastic leukaemia in the Netherlands between 1990 and 2015.

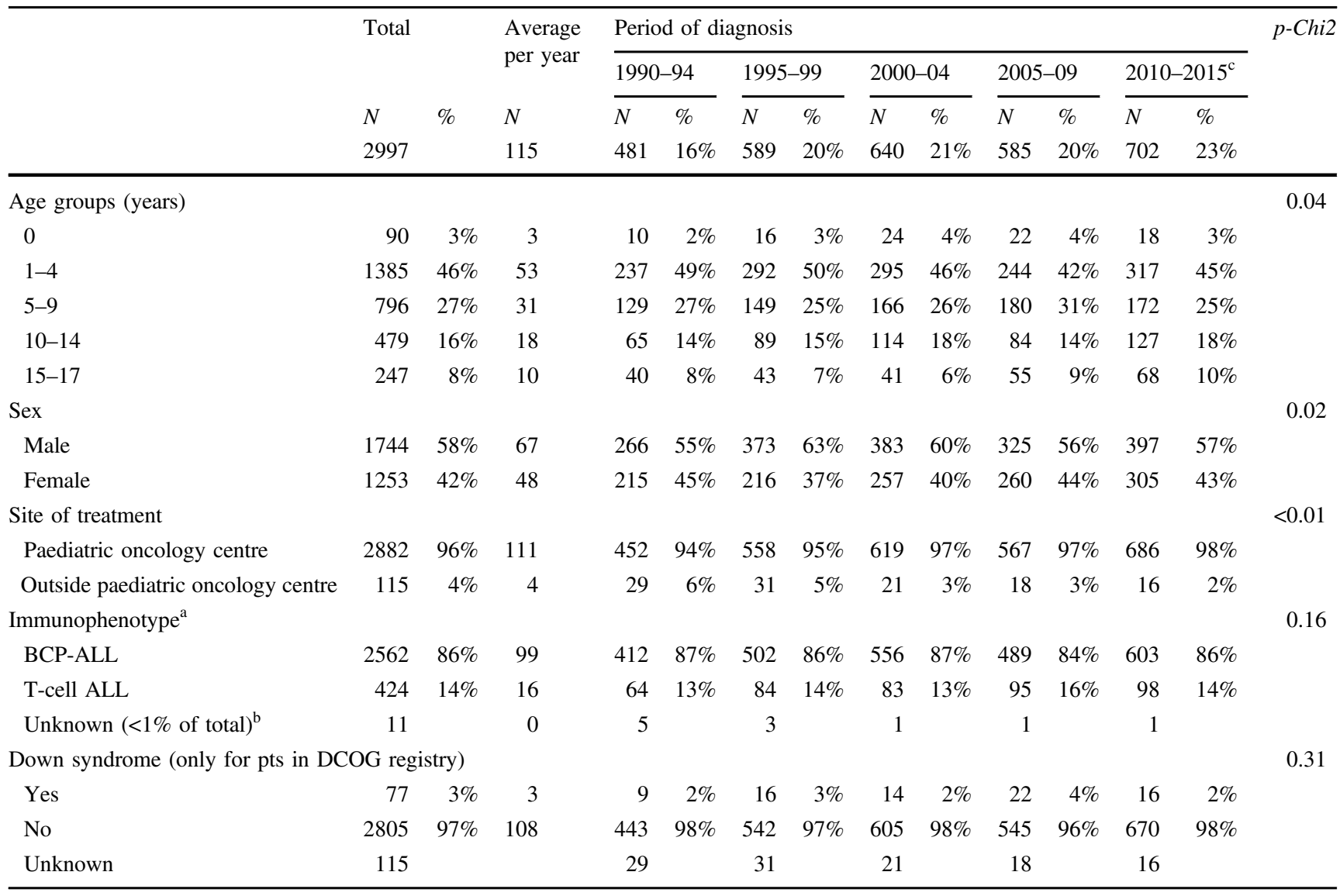

$B C P$-ALL B-cell precursor acute lymphoblastic leukaemia.

aAs confirmed by the DCOG laboratory, if not known in the DCOG registry, the NCR morphology code was taken.

${ }^{\mathrm{b}}$ Unknown if not known in the DCOG registry.

${ }^{\mathrm{c}} 6$ years period.

$1990-94$ to $93 \%$ in $2010-15(p<0.01)$ and from $77 \%$ in $1990-94$ to $89 \%$ in $2005-09(p<0.01)$, respectively. Five and 10-year overall survival for T-cell ALL did not improve (Supplementary Table S2).

\section{Determinants for risk of death}

The multivariable analysis for the risk of dying within 5years after diagnosis, adjusted for follow-up time, demonstrated a significant decrease in the hazard ratio (HR) during the periods 2005-09 and 2010-15 (HR 0.5, $p<0.01$ and HR $0.4, p<0.01$ ) compared to 1990-94 (Table 2). Infants, children aged 10-14 years and young adolescents of 15-17 years exhibited an increased risk of death compared with children of 1-4 years at diagnosis (HR 8.2, $p<0.01$, HR $2.1, p<0.01$ and HR 3.5, $p<0.01$, respectively). Patients with a T-cell ALL were at higher risk of dying compared to patients with BCP-ALL (HR 1.9, $p<0.01)$ (Table 2).

\section{Site of treatment and trends in overall survival for patients aged 15-17 year}

The percentage of patients aged 15-17 year and treated at a paediatric oncology centre increased significantly $(p<0.01)$ over time, being $87 \%(n=59)$ during 2010-15 compared with 35\% ( $n=14$ ) during 1990-94 (Fig. 4). To determine whether the site of treatment also affected outcome, we developed two multivariable analyses models. The first demonstrated a decreased risk of death over time for the two most recent periods (2005-09 HR 0.4, $p=0.03$ and 2010-15 HR 0.5, $p=04$, respectively). Addition of site of treatment, i.e. adult oncology versus paediatric oncology resulted in the loss of significance for the HRs of the recent periods of diagnosis (HR 0.6, $p=0.25$ and HR $0.8, p=$ 0.56 , respectively). In this second model, site of treatment appeared to be the most discriminative factor for reduced risk of death, i.e. an HR 0.3 for patients treated at a 
90

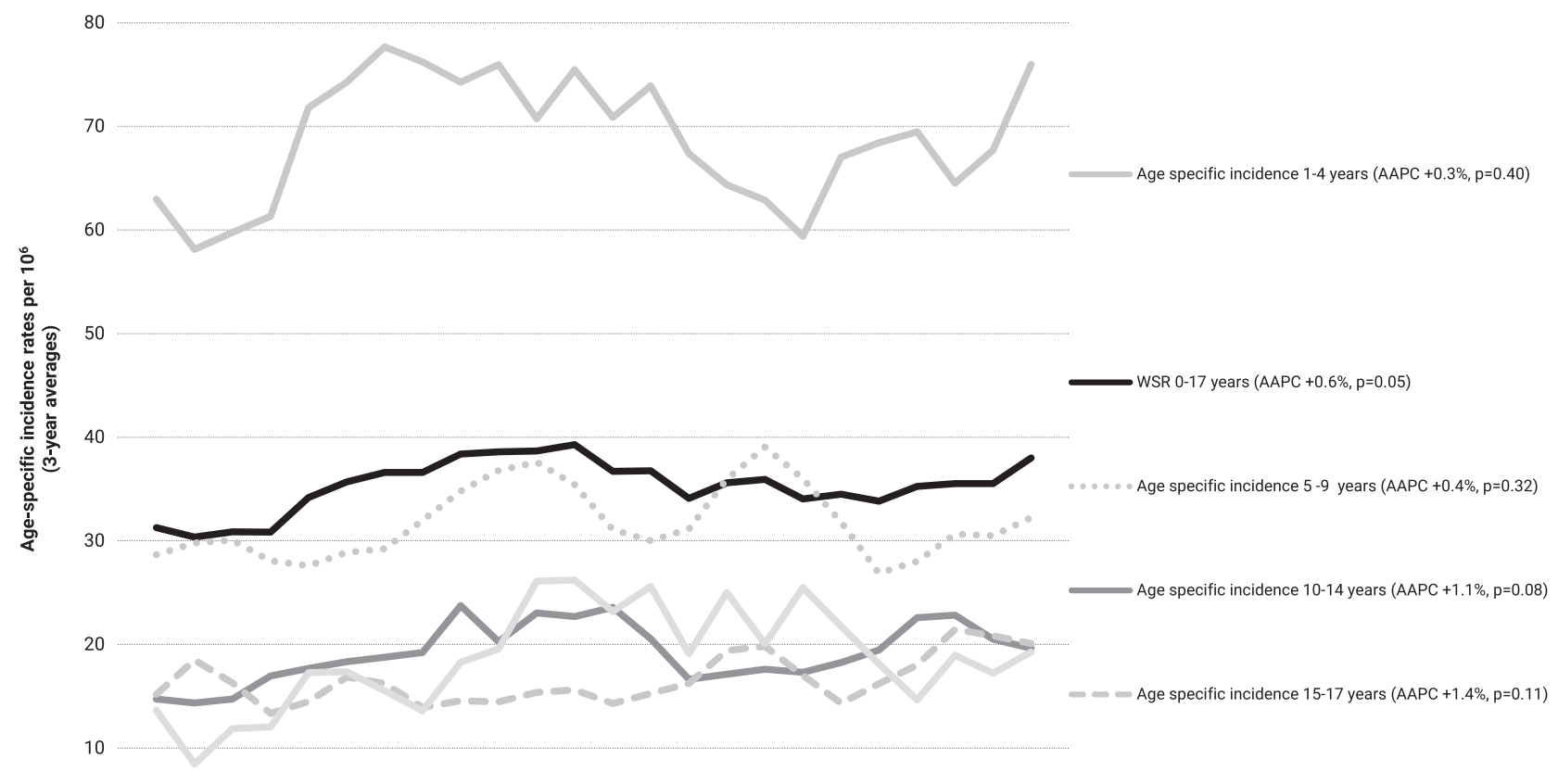

Age specific incidence 0 years (AAPC $-0.5 \%, p=0.73$ )

$\begin{array}{lllll}0 & 1995 & 2000 & 2005 & 2010\end{array}$

Fig. 1 Time trends in incidence of patients aged $<18$ years with ALL by age groups in the Netherlands, 1990-2015. Three-year moving averages of the age-standardised incidence rate of ALL (standardised according to the World Standard Rate, WSR) and age-

specific incidence rates are shown. The average annual percentage change (AAPC) was estimated for each year of diagnosis with linear regression analyses.

paediatric oncology centre compared to treatment outside a paediatric oncology centre $(p<0.01$, Table 3)

\section{Trends in mortality rates}

Mortality rates below the age of 20 years at time of death decreased remarkably from 9.5 per million children in $1980-84$ to 2.8 in $2010-16$ (a decline of $4.0 \%$ per annum, $p<0.01$ ). In the first period on average 40 young people died per year compared to 11 per year in 2010-16 (Supplementary Table S3). Also for the period 1990-2016 the AAPC trend analysis remained significant. Low numbers did not allow to observe a trend in girls below age 5 nor aged 10-14 year at death (Supplementary Table S3).

\section{Discussion}

This is the first population-based study describing trends in incidence, survival and mortality for children and adolescents aged $<18$ years with ALL in the Netherlands. Over a 26-year period we observed stable incidence rates and increasing survival rates for all ages. The progress made is

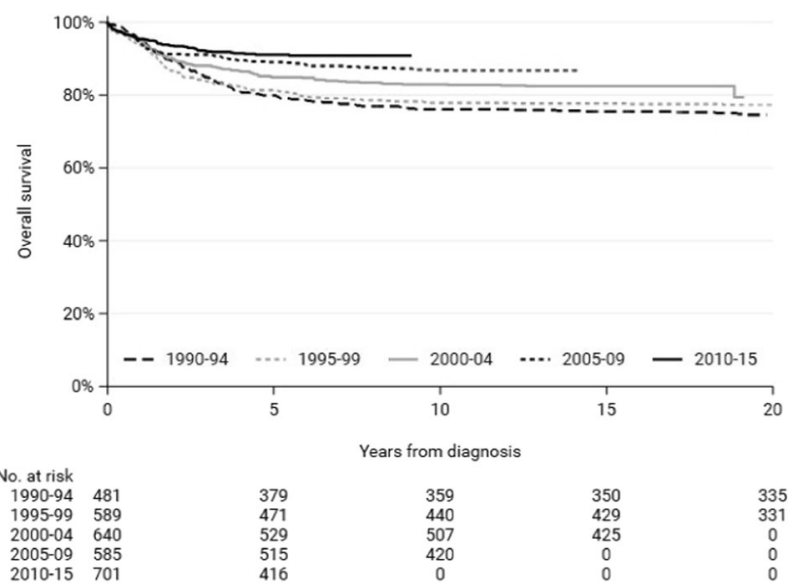

Fig. 2 Time trends in overall survival of patients aged $<\mathbf{1 8}$ years with ALL in the Netherlands, 1990-2015. The 1-year overall survival did not improve over time, $p=0.70$. The 5 - and 10 -year overall survival did improve over time, both $p<0.01$. The $p$ for trend was tested with streg and adjusted for follow-up time.

supported by steadily decreasing, independently assessed, mortality rates for all age groups. Markedly more patients of 15-17 year were treated at a paediatric oncology centre 


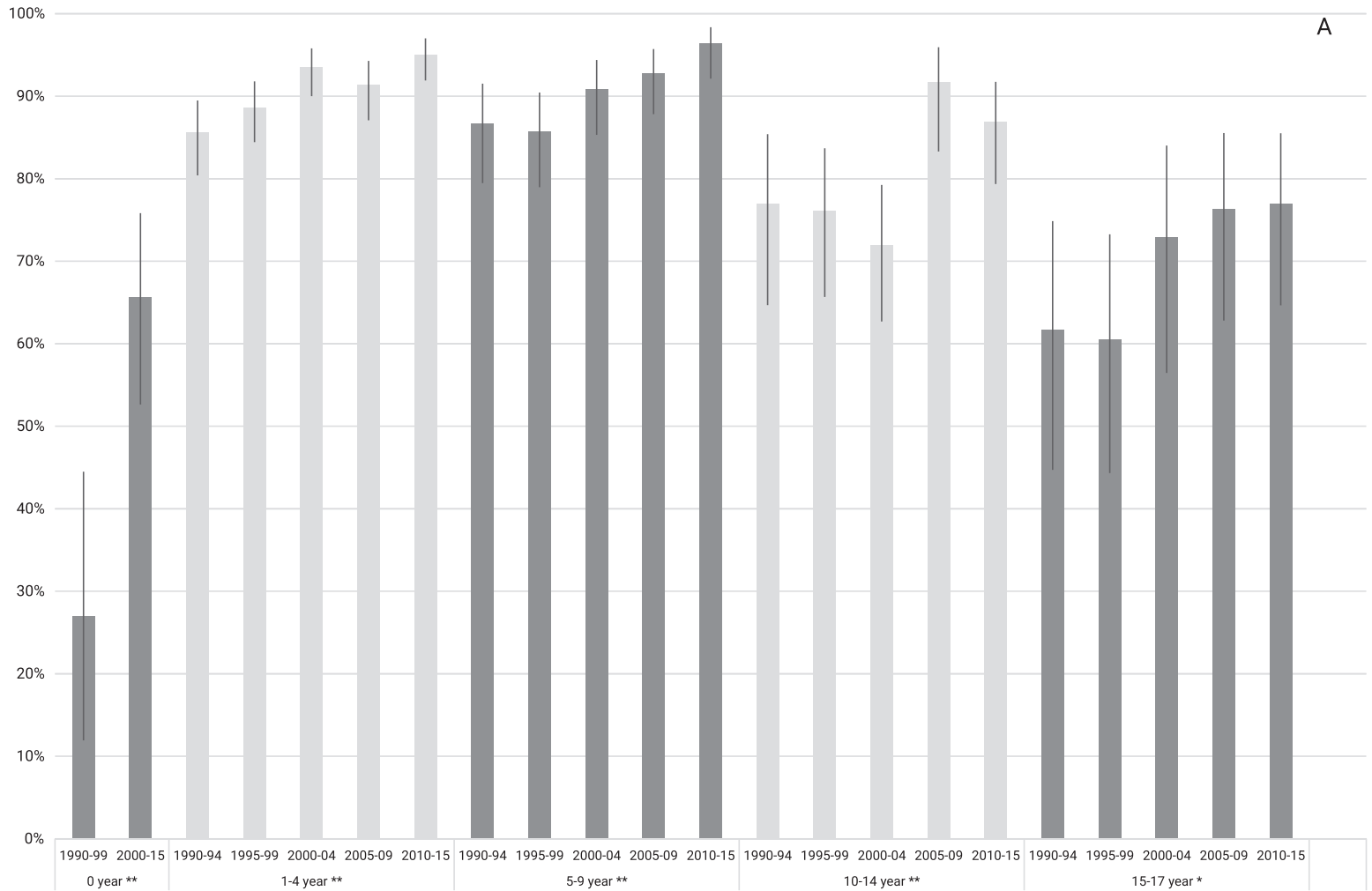

$100 \%$

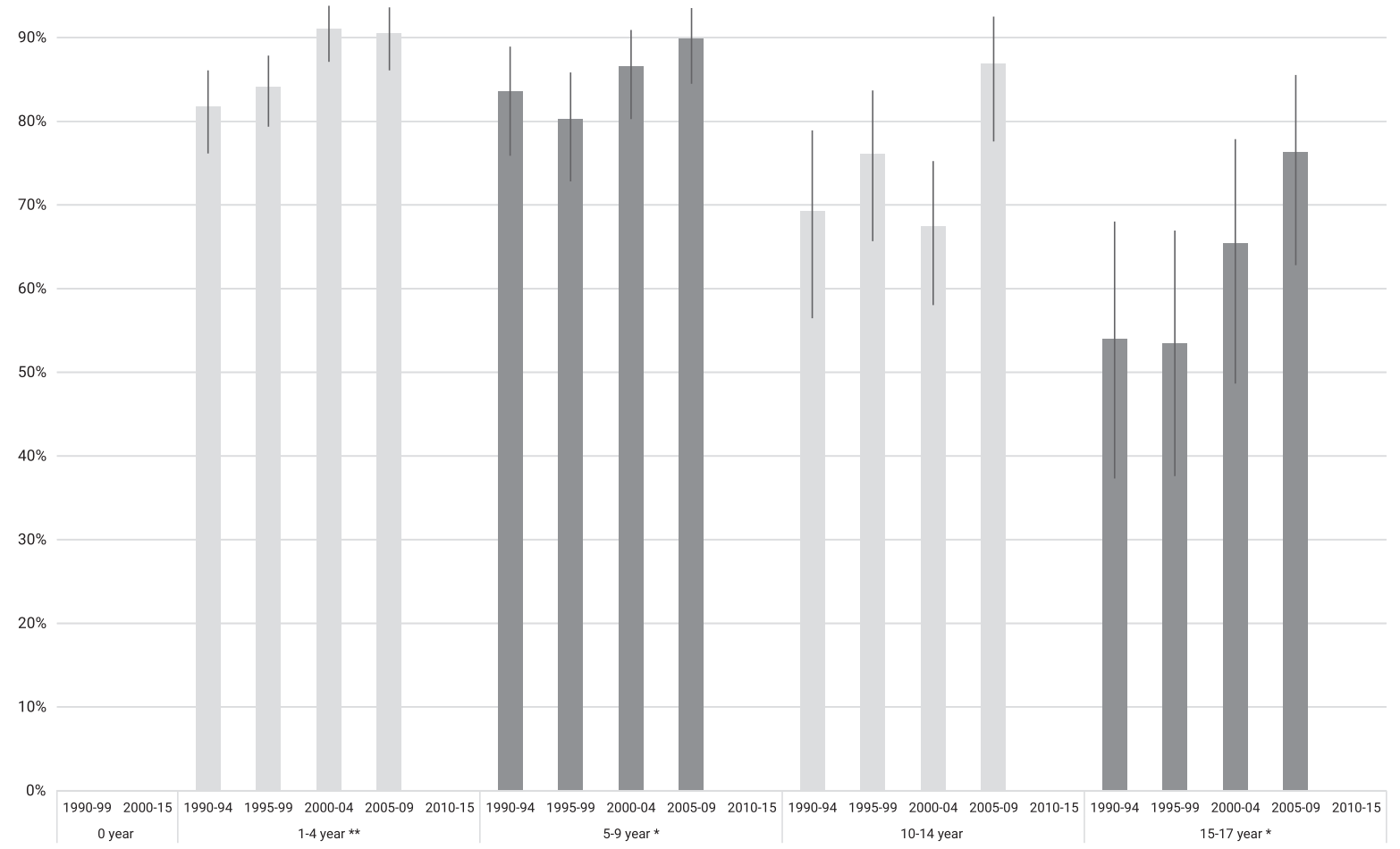


Fig. 3 Time trends in overall survival of patients aged $<18$ years with ALL by age groups in the Netherlands, 1990-2015. Five (a) and 10-year (b) overall survival with corresponding confidence intervals, corrected for follow-up time. Ten-year overall survival for infants, patients aged $<0$ years is not given due to $<20$ patients in this group. And for patients diagnosed in the last period, follow-up time is not sufficient to report 10-year survival. * Indicates significant improvement of survival over time for that age group, $p \geq 0.01$ and $p<$ $0.05 * *$ Indicates significant improvement of survival over time for that age group, $p<0.01 P$ for trend adjusted for follow-up time.

which - in a subgroup analysis - improved their outcome significantly compared with those who were not treated at a paediatric oncology centre.

The age-standardised incidence rate (WSR) of ALL increased with a modest $0.6 \%$ per year. For the last period the WSR was 37 cases per million children aged 0-17 years. This incidence rate is similar to other western countries [23, 24], although epidemiologic trend papers report mostly incidence trends for children aged $<15$ years or including adolescents $<20$ years. Compared to the reported increase in incidence in the 1990s [4, 5] we can safely assume that incidence remained almost stable after 2000. We were also able to study occurrence of BCP- or Tcell ALL specifically and notice an increase for BCP- ALL in 10-14-year-olds and for T-cell ALL in 15-17-year-olds. Although we did not correct for multiple testing, it is not rare that one or two of the results became positive, due to temporal variation. All in all, substantial influences of environmental factors, either or not pregnancy related, were unlikely to have affected risk of childhood leukaemia in the Netherlands.

Our population-based survival data demonstrated increasing rates over time, with 5-year overall survival of $80 \%$ in $1990-94$ versus $91 \%$ in $2010-15$. The populationbased study from the CONCORD working group showed similar results for patients aged 0-14 years and year of diagnosis between 1995 and 2009 for north-western European countries comparable with the Netherlands [25]. The COG has reported on the outcome of over 20,000 patients registered in their trials between 1990 and 2005, in which 5-year OS increased from 84\% in 1990-94 to $90 \%$ in 2000-05 [26] indicating very similar improvements in outcome in both North-America and Europe. Infants, older children and young adolescents had a less favourable prognosis compared to children aged 1-9 years. This might be explained by the higher incidence of unfavourable features such as KMT2A rearrangements [19] in infants and a higher incidence of BCR-ABL like abnormalities [27] and lower incidence of favourable prognostic features such as ETV6-RUNX1 and hyperdiploidy in older patients [12]. The increase in survival rate from $27 \%$ in $1990-99$ to $66 \%$ in
Table 2 Multivariable analysis for the risk of dying from acute lymphoblastic leukaemia for patients aged $<18$ years in the Netherlands between 1990 and 2015.

\begin{tabular}{|c|c|c|c|c|}
\hline & $N$ & $\mathrm{HR}^{\mathrm{a}}$ & $95 \% \mathrm{CI}$ & $P$ value \\
\hline \multicolumn{5}{|l|}{ Period } \\
\hline 1990-94 & 481 & Ref. & & \\
\hline 1995-99 & 589 & 0.9 & $0.7-1.2$ & 0.56 \\
\hline 2000-04 & 640 & 0.7 & $0.5-0.9$ & 0.01 \\
\hline 2005-09 & 585 & 0.5 & $0.1-0.3$ & $<0.01$ \\
\hline $2010-15$ & 702 & 0.4 & $0.1-0.3$ & $<0.01$ \\
\hline \multicolumn{5}{|l|}{ Sex } \\
\hline Male & 1744 & Ref. & & \\
\hline Female & 1253 & 0.9 & $0.7-1.1$ & 0.19 \\
\hline \multicolumn{5}{|c|}{ Age groups (years) } \\
\hline 0 & 90 & 8.2 & $5.8-12$ & $<0.01$ \\
\hline $1-4$ & 1385 & Ref. & & \\
\hline $5-9$ & 796 & 1 & $0.8-1.4$ & 0.79 \\
\hline $10-14$ & 479 & 2.1 & $1.6-2.8$ & $<0.01$ \\
\hline $15-17$ & 247 & 3.5 & $2.6-4.7$ & $<0.01$ \\
\hline \multicolumn{5}{|c|}{ Immunophenotype } \\
\hline BCP-ALL & 2562 & Ref. & & \\
\hline T-cell ALL & 424 & 1.9 & $1.5-2.4$ & $<0.01$ \\
\hline Unknown & 11 & ND & & \\
\hline
\end{tabular}

$B C P$-ALL B-cell precursor acute lymphoblastic leukaemia, $H R$ hazard ratio, $C I$ confidence interval, $N D$ not done.

${ }^{\text {a } I n ~ t h i s ~ m u l t i v a r i a b l e ~ a n a l y s i s, ~ e a c h ~ c o v a r i a t e ~ i s ~ s i m u l t a n e o u s l y ~}$ adjusted for all other covariates and follow-up time. Hazard ratios represent risk of death within 5 years from diagnosis compared to the reference category.

2000-2015 in infants is likely due to the implementation of the Interfant treatment schemes including more intensive use of cytosine arabinoside [18, 19]. It should be mentioned that the confidence intervals for infants are broad due to small numbers. Also, 5-year survival rate of $80 \%$ for T-cell ALL in 2010-15 was lower than the 93\% for BCP-ALL. Historically, T-ALL patients have had a worse prognosis than other ALL patients $[12,13,28]$. With the better treatment stratifications based on MRD, the outcome for T-ALL patients improved to $81 \%$ in $2010-15$ but there is still a gap with B-lineage ALL.

Five and 10-year overall survival rates for adolescents aged 15-17 years increased from <60\% in 1990-94 to $\sim 75 \%$ in $2010-15$. The better hospital-based survival rates for adolescents (and young adults) were attained when adolescents were treated on paediatric ALL protocols compared to adult protocols about 15 years ago [29-31]. The percentage of patients aged 15-17 years treated at a paediatric oncology centre increased over time from $35 \%$ to $87 \%$ in the past 25 years in our study. Interestingly, a 


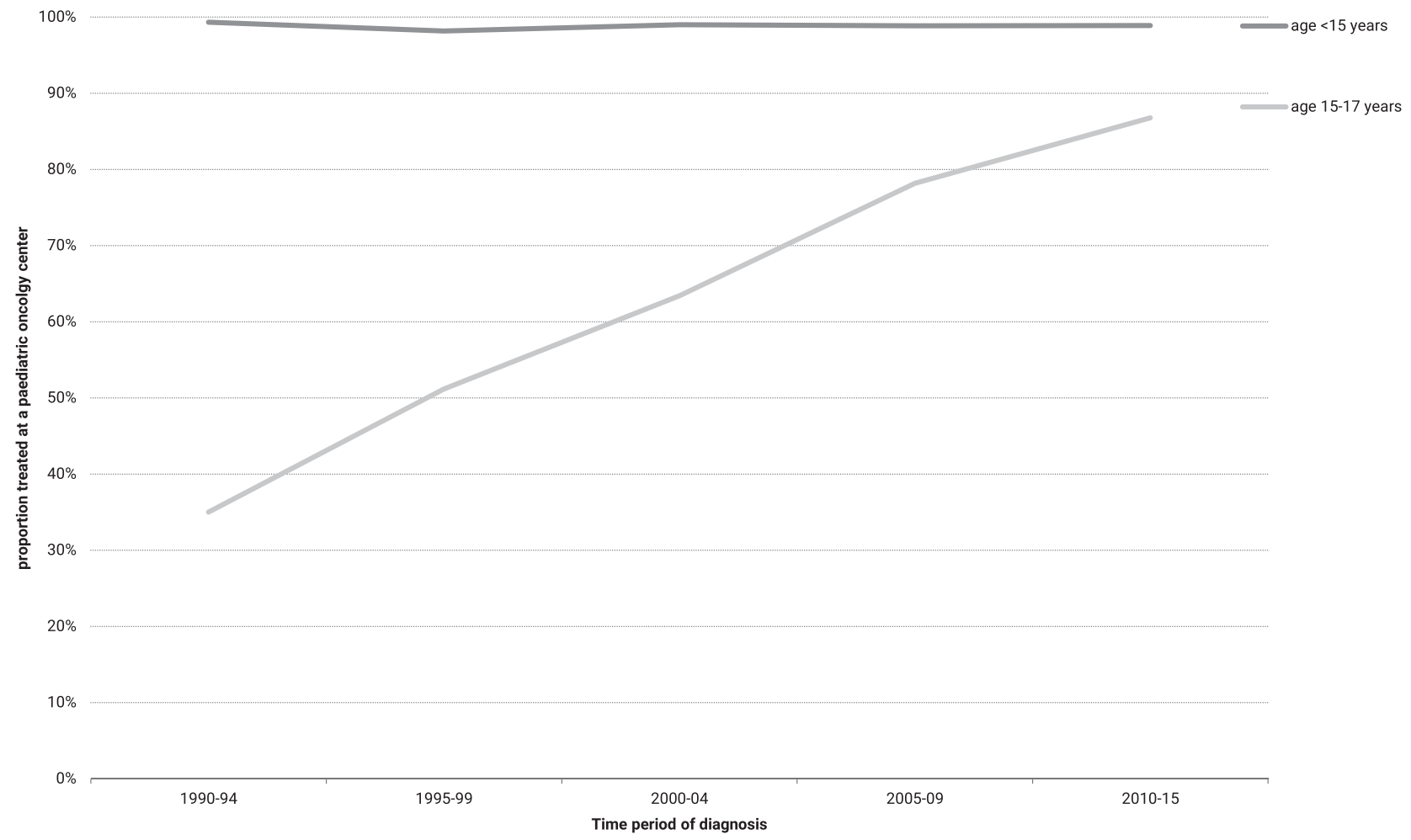

Fig. 4 Proportion of patients with ALL treated at a paediatric oncology centre by age groups, 1990-2015. Age groups are displayed in dark gray as age $<15$ years and light gray age $15-17$ years, respectively.

multivariable analysis showed that treatment of patients aged 15-17 years in a paediatric oncology centre led to a better outcome. Since the early 2000s young adult ALL treatment protocols have been adapted to the more paediatric like treatment approaches with dose-intensity of nonmyelotoxic therapies and stricter timing of subsequent courses [32]. Possibly, there are still differences in management of treatment-related toxicities and/or trial participation in adult versus paediatric centres [33].

In agreement with other studies, mortality rates declined constantly over time at each age group [34, 35]. Increased intensity of induction and reinduction therapy were the first important components of successful ALL treatment protocols at the end of the 1970s and 1980s [36]. We could not report on the incidence and survival in the 1980s because this was before initiation of the NCR. Improvements in chemotherapy and better ways to stratify patients based upon genetic abnormalities and on initial treatment response measured by minimal residual disease [18-20], together with specific protocols for infants and BCR-ABL positive patients, further improved outcome for ALL patients. Supplementary Table 4 shows outcome data of the DCOG protocols used during the time period of the present study. There was no change in death before remission or death in remission over time. The improved survival has been achieved by better initial treatments leading to significantly improved EFS (from 66\% to 89\%) but part of patients is still rescued by relapse therapy illustrated by the gap between EFS and OS. The rate of stem cell transplantation did not significantly change over time. The proportion of secondary malignancies is below $2 \%$ on all DCOG protocols in the time period of the present study $[12,37]$.

Although detailed information on treatment schemes (initial and relapse treatment), risk group or response status are lacking in the NCR for individual patients, we did not have the intention with this descriptive epidemiological study to study outcome by treatment protocol or risk group. We just wanted to show whether there was progress. Strengths of our study include the linkage with the DCOG clinical registry over the whole study period. We could thus obtain morphology codes of almost all patients by centralised expert haemato-pathology review and determine the proportion of patients treated in a paediatric oncology centre. The latter improvement may be a stimulus for other groups.

All in all, by combining incidence, survival and mortality data we attained a comprehensive picture of the progress against ALL in children and young adolescents in the Netherlands by showing improved survival, especially 
Table 3 Univariate and multivariable analyses for the risk of dying from acute lymphoblastic leukaemia for patients aged $15-17$ years in the Netherlands between 1990 and 2015.

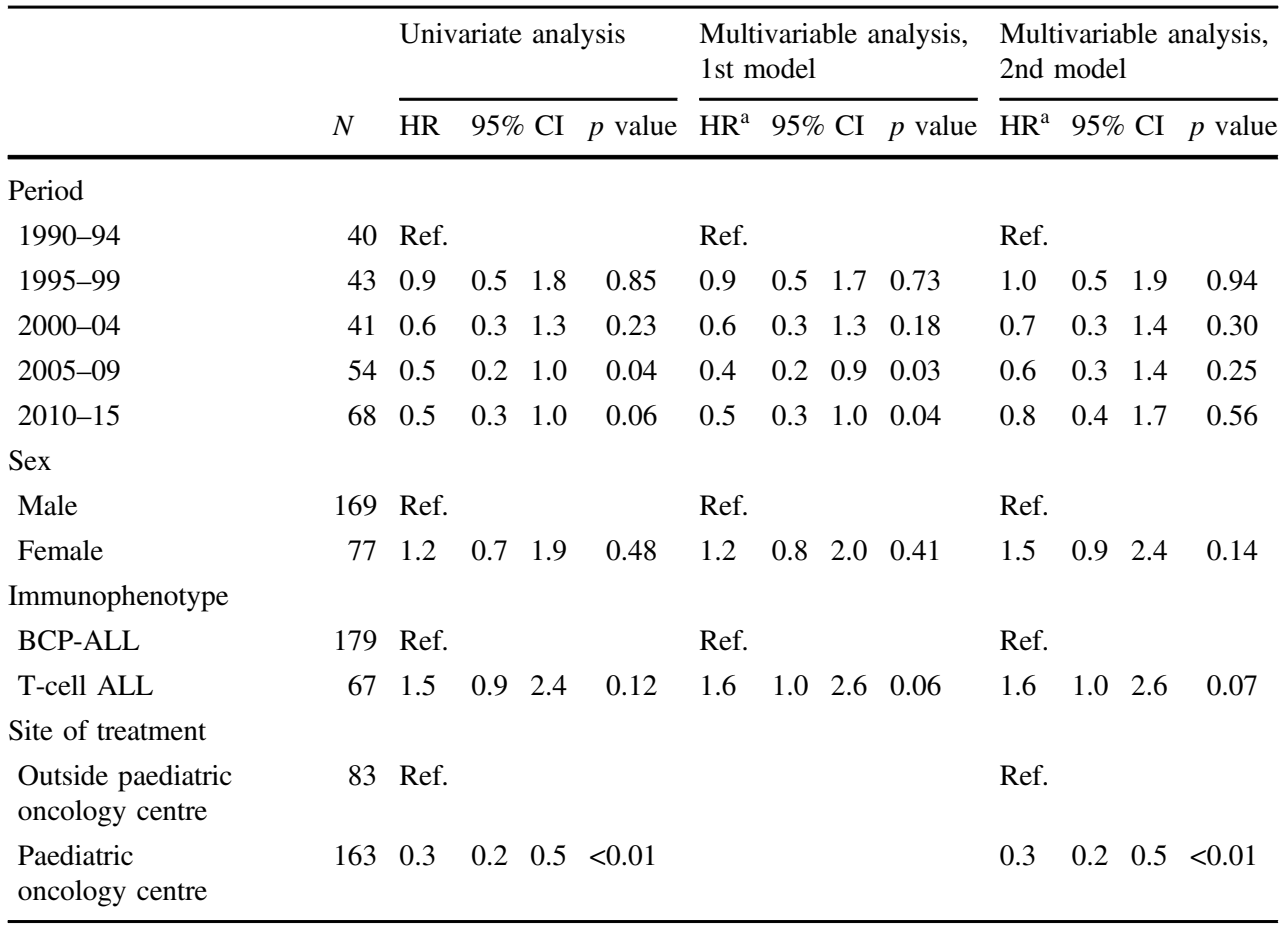

In the first multivariable model we did not consider site of treatment, and this model shows significantly lower risk of death in recent periods of diagnosis compared to the reference period 1990-1994. In the second multivariable model we added site of treatment which results in disappearance of the discriminative effect of period of diagnosis and a significantly lower risk of death for patients treated in a paediatric oncology centre. $B C P$ - $A L L$ B-cell precursor acute lymphoblastic leukaemia, $H R$ hazard ratio, $C I$ confidence interval.

${ }^{a}$ In the multivariable analysis, each covariate is simultaneously adjusted for all other covariates, and followup time. Hazard ratios represent risk of death within 5 years from diagnosis compared to the reference category.

improved survival of adolescents treated in a paediatric oncology centre, and supported by steadily declining mortality rates. The overall incidence rate was stable, despite two age and type-specific increases.

Acknowledgements The current work is funded by Stichting Kinderen Kankervrij (KiKa) [project number 207]. The funding source had no role in the study design, data collection, analyses and interpretation of the results, nor in writing of this manuscript. We thank the registration team of the Netherlands Comprehensive Cancer Organization (IKNL) for the collection of data for the Netherlands Cancer Registry. We thank Dr. Otto Visser (IKNL) for the extra data checks for the current study. We thank the laboratory personnel and the data managers from the Dutch Childhood Oncology Group (DCOG) for collecting the data for the DCOG registry. We recognise all paediatric oncologists who treated the patients and provided data in the DCLSG/DCOG collaboration.

Author contributions AMJR, JWWC, HAdGK, LCK and RP contributed to the study design. HAdGK and HEKK contributed to data acquisition. AMJR analysed the data. AMJR, HAdGK, JWWC, IMvdS, LCK, HEKK and RP interpreted the results. AMJR, HEKK and RP draughted and AMJR, HAdGK, JWWC, IMvdS, LCK, HEKK and RP revised the manuscript. All authors approved the final version of the manuscript. AR had full access to all the data in the study and had final responsibility for the decision to submit for publication.

\section{Compliance with ethical standards}

Conflict of interest The authors declare that they have no conflict of interest.

Publisher's note Springer Nature remains neutral with regard to jurisdictional claims in published maps and institutional affiliations.

Open Access This article is licensed under a Creative Commons Attribution 4.0 International License, which permits use, sharing, adaptation, distribution and reproduction in any medium or format, as long as you give appropriate credit to the original author(s) and the source, provide a link to the Creative Commons license, and indicate if changes were made. The images or other third party material in this article are included in the article's Creative Commons license, unless indicated otherwise in a credit line to the material. If material is not included in the article's Creative Commons license and your intended use is not permitted by statutory regulation or exceeds the permitted use, you will need to obtain permission directly from the copyright holder. To view a copy of this license, visit http://creativecommons. org/licenses/by/4.0/.

\section{References}

1. Xie Y, Davies SM, Xiang Y, Robison LL, Ross JA. Trends in leukemia incidence and survival in the United States (1973-1998). Cancer. 2003;97:2229-35. 
2. Dreifaldt AC, Carlberg M, Hardell L. Increasing incidence rates of childhood malignant diseases in Sweden during the period 1960-1998. Eur J Cancer. 2004;40:1351-60.

3. Steliarova-Foucher E, Stiller C, Kaatsch P, Berrino F, Coebergh JW, Lacour B, et al. Geographical patterns and time trends of cancer incidence and survival among children and adolescents in Europe since the 1970s (the ACCISproject): an epidemiological study. Lancet. 2004;364:2097-105.

4. Reedijk AM, Janssen-Heijnen ML, Louwman MW, Snepvangers Y, Hofhuis WJ, Coebergh JW. Increasing incidence and improved survival of cancer in children and young adults in Southern Netherlands, 1973-1999. Eur J Cancer. 2005;41:760-9.

5. Coebergh JW, Reedijk AM, de Vries E, Martos C, Jakab Z, Steliarova-Foucher E, et al. Leukaemia incidence and survival in children and adolescents in Europe during 1978-1997. Report from the Automated Childhood Cancer Information System project. Eur J Cancer. 2006;42:2019-36.

6. Smith MA, Seibel NL, Altekruse SF, Ries LA, Melbert DL, O'Leary M, et al. Outcomes for children and adolescents with cancer: challenges for the twenty-first century. J Clin Oncol. 2010;28:2625-34.

7. Hunger SP, Mullighan CG. Acute lymphoblastic leukemia in children. N. Engl J Med. 2015;373:1541-52.

8. Karim-Kos HE, Kiemeney LA, Louwman MW, Coebergh JW, de Vries E. Progress against cancer in the Netherlands since the late 1980s: an epidemiological evaluation. Int $\mathbf{J}$ Cancer. 2012;130:2981-9.

9. Kamps WA, Bokkerink JP, Hahlen K, Hermans J, Riehm H, Gadner $\mathrm{H}$, et al. Intensive treatment of children with acute lymphoblastic leukemia according to ALL-BFM-86 without cranial radiotherapy: results of Dutch Childhood Leukemia Study Group Protocol ALL-7 (1988-1991). Blood. 1999;94:1226-36.

10. Kamps WA, Bokkerink JP, Hakvoort-Cammel FG, Veerman AJ, Weening RS, van Wering ER, et al. BFM-oriented treatment for children with acute lymphoblastic leukemia without cranial irradiation and treatment reduction for standard risk patients: results of DCLSG protocol ALL-8 (1991-1996). Leukemia. 2002;16: 1099-111.

11. Veerman AJ, Kamps WA, van den Berg H, van den Berg E, Bokkerink JP, Bruin MC, et al. Dexamethasone-based therapy for childhood acute lymphoblastic leukaemia: results of the prospective Dutch Childhood Oncology Group (DCOG) protocol ALL-9 (1997-2004). Lancet Oncol. 2009;10:957-66.

12. Pieters R, de Groot-Kruseman H, Van der Velden V, Fiocco M, van den Berg H, de Bont E, et al. Successful therapy reduction and intensification for childhood acute lymphoblastic leukemia based on minimal residual disease monitoring: study ALL10 from the Dutch childhood oncology group. J Clin Oncol. 2016;34: 2591-601.

13. Pui CH, Yang JJ, Hunger SP, Pieters R, Schrappe M, Biondi A, et al. Childhood acute lymphoblastic leukemia: progress through collaboration. J Clin Oncol. 2015;33:2938-48.

14. Pastore G, Viscomi S, Gerov GL, Terracini B, Madon E, Magnani C. Population-based survival after childhood lymphoblastic leukaemia in time periods corresponding to specific clinical trials from 1979 to 1998-a report from the Childhood Cancer Registry of Piedmont (Italy). Eur J Cancer. 2003;39:952-60.

15. Stiller CA, Kroll ME, Pritchard-Jones K. Population survival from childhood cancer in Britain during 1978-2005 by eras of entry to clinical trials. Ann Oncol. 2012;23:2464-9.

16. World Health Organisation. International classification of diseases for oncology (ICD-O), 1st revision. 3rd ed. Geneva: World Health Organisation; 2013.

17. van der Sanden GA, Coebergh JW, Schouten LJ, Visser O, van Leeuwen FE. Cancer incidence in The Netherlands in 1989 and 1990: first results of the nationwide Netherlands cancer registry.
Coordinating Committee for Regional Cancer Registries. Eur J Cancer. 1995;31A:1822-9.

18. Pieters R, Schrappe M, De Lorenzo P, Hann I, De Rossi G, Felice $\mathrm{M}$, et al. A treatment protocol for infants younger than 1 year with acute lymphoblastic leukaemia (Interfant-99): an observational study and a multicentre randomised trial. Lancet. 2007;370: 240-50.

19. Pieters R, De Lorenzo P, Ancliffe P, Aversa LA, Brethon B, Biondi A, et al. Outcome of infants younger than 1 year with acute lymphoblastic leukemia treated with the interfant-06 protocol: results from an international phase iii randomized study. J Clin Oncol. 2019;37:2246-56.

20. Biondi A, Schrappe M, De Lorenzo P, Castor A, Lucchini G, Gandemer V, et al. Imatinib after induction for treatment of children and adolescents with Philadelphia-chromosome-positive acute lymphoblastic leukaemia (EsPhALL): a randomised, openlabel, intergroup study. Lancet Oncol. 2012;13:936-45.

21. Boyle P, Parkin DM. Statistical methods for registries. Cancer registration: principles and methods. Lyon: IARC; 1991. pp. 126-58.

22. Clegg LX, Hankey BF, Tiwari R, Feuer EJ, Edwards BK. Estimating average annual per cent change in trend analysis. Stat Med. 2009;28:3670-82.

23. Linet MS, Brown LM, Mbulaiteye SM, Check D, Ostroumova E, Landgren $\mathrm{A}$, et al. International long-term trends and recent patterns in the incidence of leukemias and lymphomas among children and adolescents ages 0-19 years. Int J Cancer. 2016; 138:1862-74.

24. Baade PD, Youlden DR, Valery PC, Hassall T, Ward L, Green $\mathrm{AC}$, et al. Trends in incidence of childhood cancer in Australia, 1983-2006. Br J Cancer. 2010;102:620-6.

25. Bonaventure A, Harewood R, Stiller CA, Gatta G, Clavel J, Stefan DC, et al. Worldwide comparison of survival from childhood leukaemia for 1995-2009, by subtype, age, and sex (CONCORD2): a population-based study of individual data for 89828 children from 198 registries in 53 countries. Lancet Haematol. 2017;4: e202-17.

26. Hunger SP, Lu X, Devidas M, Camitta BM, Gaynon PS, Winick $\mathrm{NJ}$, et al. Improved survival for children and adolescents with acute lymphoblastic leukemia between 1990 and 2005: a report from the children's oncology group. J Clin Oncol. 2012;30: 1663-9.

27. Den Boer ML, van Slegtenhorst M, De Menezes RX, Cheok MH, Buijs-Gladdines JG, Peters ST, et al. A subtype of childhood acute lymphoblastic leukaemia with poor treatment outcome: a genomewide classification study. Lancet Oncol. 2009;10:125-34.

28. Muffly L, Larson RA. Improving outcomes in childhood T-cell acute lymphoblastic leukemia: promising results from the Children's Oncology Group incorporating nelarabine into front-line therapy. Transl Pediatr. 2012;1:120-2.

29. Boissel N, Auclerc MF, Lheritier V, Perel Y, Thomas X, Leblanc $\mathrm{T}$, et al. Should adolescents with acute lymphoblastic leukemia be treated as old children or young adults? Comparison of the French FRALLE-93 and LALA-94 trials. J Clin Oncol. 2003;21: $774-80$.

30. de Bont JM, Holt B, Dekker AW, van der Does-van den Berg A, Sonneveld P, Pieters R. Significant difference in outcome for adolescents with acute lymphoblastic leukemia treated on pediatric vs adult protocols in the Netherlands. Leukemia. 2004;18: 2032-5.

31. Stock W, La M, Sanford B, Bloomfield CD, Vardiman JW, Gaynon P, et al. What determines the outcomes for adolescents and young adults with acute lymphoblastic leukemia treated on cooperative group protocols? A comparison of Children's Cancer Group and Cancer and Leukemia Group B studies. Blood. 2008;112:1646-54. 
32. Dinmohamed AG, Szabo A, van der Mark M, Visser O, Sonneveld $\mathrm{P}$, Cornelissen JJ, et al. Improved survival in adult patients with acute lymphoblastic leukemia in the Netherlands: a population-based study on treatment, trial participation and survival. Leukemia. 2016;30:310-7.

33. Siegel SE, Advani A, Seibel N, Muffly L, Stock W, Luger S, et al. Treatment of young adults with Philadelphia-negative acute lymphoblastic leukemia and lymphoblastic lymphoma: hyperCVAD vs. pediatric-inspired regimens. Am J Hematol. 2018;93:1254-66.

34. Levi F, Lucchini F, Negri E, La Vecchia C. Trends in cancer mortality at age 15 to 24 years in Europe. Eur $\mathrm{J}$ Cancer. 2003;39:2611-21.
35. Bosetti C, Bertuccio P, Chatenoud L, Negri E, Levi F, La Vecchia C. Childhood cancer mortality in Europe, 1970-2007. Eur J Cancer. 2010;46:384-94.

36. Reiter A, Schrappe M, Ludwig WD, Hiddemann W, Sauter S, Henze G, et al. Chemotherapy in 998 unselected childhood acute lymphoblastic leukemia patients. Results and conclusions of the multicenter trial ALL-BFM 86. Blood. 1994;84:3122-33.

37. Kamps WA, van der Pal-de Bruin KM, Veerman AJ, Fiocco M, Bierings M, Pieters R. Long-term results of Dutch Childhood Oncology Group studies for children with acute lymphoblastic leukemia from 1984 to 2004. Leukemia. 2010;24: 309-19. 